Portland State University

PDXScholar

$1-1-1996$

\title{
Generalized Beam Matrices. II. Mode Selection in Lasers and Periodic Misaligned Complex Optical Systems
}

Lee W. Casperson

Portland State University

Anthony A. Tovar

Follow this and additional works at: https://pdxscholar.library.pdx.edu/ece_fac

Part of the Electrical and Computer Engineering Commons Let us know how access to this document benefits you.

\section{Citation Details}

Anthony A. Tovar and Lee W. Casperson, "Generalized beam matrices. II. Mode selection in lasers and periodic misaligned complex optical systems," J. Opt. Soc. Am. A 13, 90-96 (1996).

This Article is brought to you for free and open access. It has been accepted for inclusion in Electrical and Computer Engineering Faculty Publications and Presentations by an authorized administrator of PDXScholar. Please contact us if we can make this document more accessible: pdxscholar@pdx.edu. 


\title{
Generalized beam matrices. II. Mode selection in lasers and periodic 'misaligned complex optical systems
}

\author{
Anthony A. Tovar* and Lee W. Casperson \\ Department of Electrical Engineering, Portland State University, Portland, Oregon 97207-0751 \\ Received April 19, 1995; revised manuseript received August 1, 1995; accepted August 1, 1995
}

\begin{abstract}
A generalized beam matrix method is used to investigate the mode structure of astigmatic misaligned optical systems with loss or gain. In these optical systems the usual real-argument polynomial-Gaussian beams are not eigenfunctions, and off-axis complex-argument polynomial beams must be used. New beam transformations for these complex-argument modes are reported. Stability criteria are developed, and mode selection in laser resonators that contain tilted, displaced, or curved complex optical elements is discussed. (C) 1996 Optical Society of America
\end{abstract}

\section{INTRODUCTION}

A complex optical system is a sequence of optical elements in which one or more of the elements provides gain or loss to an input light beam. Gaussian transmission filters (and Gaussian variable-reflectivity mirrors), ${ }^{1-6}$ exponential transmission filters (and exponential variable-reflectivity mirrors), ${ }^{7}$ homogeneous amplifiers and absorbers, ${ }^{8}$ amplifiers (and absorbers) with a linear gain (loss) profile, ${ }^{7,9}$ and amplifiers (and absorbers) with a quadratic gain (loss) profile ${ }^{10-12}$ are all complex optical elements. Amplifiers and absorbers may be in the shape of a wedge or a lens. ${ }^{7}$ A medium that may have both quadratic gain and refractive index profiles is known as a complex lenslike medium, ${ }^{11}$ and a similar linearly profiled medium is a complex prismlike medium. ${ }^{7}$

Complex optical elements are often used in modern optical design. Gaussian variable-reflectivity mirrors have been used in $\mathrm{CO}_{2}$ (Refs. 13-16) and Nd:YAG (Refs. 17-21) lasers for lidar (light detection and ranging ${ }^{22}$ and high-power applications. High diffraction loss (unstable resonator) lasers are stable against temperature fluctuations, external vibrations and other mechanical perturbations, ${ }^{21}$ and mode-media instabilities. ${ }^{23}$ Resonators with Gaussian variable-reflectivity mirrors have several of the advantages of conventional highdiffraction-loss resonators without suffering from poor mode quality. In the past a disadvantage of variablereflectivity mirrors has been their poor power-handling characteristics. However, increased interest in the field has spawned several novel variable-reflectivity mirror designs that are simple and have a high damage threshold. ${ }^{24}$

To some extent, every laser possesses some sort of gain profile and is thus a complex optical system. This effect is well documented for gas lasers, which inevitably have a radial current distribution and thus a radial gain profile. ${ }^{25}$ Lasers that are optically pumped with a nonuniform field such as a Gaussian beam also possess nonuniform gain distributions. ${ }^{26}$ Absorption in the laser medium also leads to an inhomogeneous gain profile in optically pumped lasers. Gain profiles are also present in solid and liquid lasers with nonuniform ion doping, or dye molarity. Many semiconductor lasers also possess transverse gain distributions. The subject of gainprofiled media has been recently reviewed. ${ }^{27}$ Though the existence of nonuniform transverse gain in laser amplifiers is often inevitable, the effect may sometimes be exploited in modern optical systems design. For example, in high-gain $\mathrm{Xe}$ and $\mathrm{He}-\mathrm{Xe}$ lasers ${ }^{11}$ and subsequently in double heterostructure GaAs lasers, ${ }^{28}$ the transverse gain profile has been used to simplify resonator design.

Lasers with Gaussian variable-reflectivity mirrors and transverse gain profiles are only two examples of the plethora of complex Gaussian beam optical systems. Omnipresent misalignment effects in these systems have long been of interest, as minute misalignments can have drastic effects on a laser's output. ${ }^{29}$ Previously, numerical methods have been used to examine misalignment effects. Analytical techniques have also been of interest since it was found that a Gaussian beam is an eigenmode even of misaligned complex optical systems, and solutions were found with the use of a complicated diffraction integral on an ad hoc basis. ${ }^{29,30}$ The analysis was later generalized to include higher-order modes. ${ }^{31}$ Recently, a systematic method has been developed to examine the propagation of fundamental Gaussian beams in misaligned complex optical systems by use of simple matrix multiplication. ${ }^{7}$ The purpose of this paper is to extend this generalized beam matrix method to include transformations for higher-order polynomial-Gaussian modes and to investigate mode selection in misaligned complex optical systems.

In Section 2 the essential aspects of the generalized beam matrix theory is reviewed, and a new transformation for polynomial-Gaussian beams is obtained. In Section 3 important details of resonator mode structure are obtained by the application of oscillation conditions to the polynomial-Gaussian beam transformations. Mathematical conditions for confinement and perturbation stability are also investigated. In Section 4 we use the results to examine mode selection in lasers and periodic misaligned complex optical systems. 


\section{GAUSSIAN BEAM THEORY}

The generalized beam matrix formalism applies to linear media with spatially varying gain (or loss), $\alpha(x, y, z)$, and/or index of refraction, $n(x, y, z)$, of the form

$$
\begin{aligned}
k(x, y, z) \equiv & \frac{2 \pi n(x, y, z)}{\lambda}+i \alpha(x, y, z) \\
= & k_{0}(z)-k_{1 x}(z) x / 2-k_{1 y}(z) y / 2-k_{2 x}(z) x^{2} / 2 \\
& -k_{2 y}(z) y^{2} / 2
\end{aligned}
$$

There are three explicit approximations made in the theory: the scalar approximation, the paraxial approximation, and the slowly varying quadratic-profile approximation. The neglect of nonlinear effects such as saturation is assumed implicitly. Similarly, the assumption of an ideal quadratic propagation constant or phase variation can be valid only out to some finite radius in a realizable medium. It is therefore also assumed that the propagating modes are completely confined within the quadratic-profile region. Though not all profiled media of interest are parabolic, the quadratic is an excellent approximation near the center of any smoothly peaked function, including the Bessel radial dependence that occurs in many gas lasers ${ }^{25}$ and a Gaussian radial dependence that is due to optical pumping or similar profiles that are due to transverse spatial hole burning. ${ }^{26} \mathrm{~A}$ fourth explicit approximation that is usually made is the low-gain-per-wavelength approximation. Though this is an acceptable approximation for many optical systems of interest, it is unnecessary and makes the formalism no easier. ${ }^{8}$ Therefore this approximation is not made here.

With these approximations the eigenmodes for any medium described by Eq. (2) and several thin optical elements such as a lens, a prism, a Gaussian aperture, and an exponential aperture can be written as

$$
\begin{aligned}
\bar{E}_{m n}(x, y, z, t)= & \operatorname{Re}\left(E_{m n}^{\prime}(x, y, z)\right. \\
& \left.\times \exp \left\{i\left[\omega t-\int_{0}^{z} k_{0}\left(z^{\prime}\right) \mathrm{d} z^{\prime}\right]\right\}\right) \\
& \times\left[\cos (\nu) \bar{i}_{x}+\sin (\nu) \bar{i}_{y}\right],
\end{aligned}
$$

where the complex electric-field factor is

$$
\begin{aligned}
E_{m n}^{\prime}(x, y, z)= & E_{m n, 0}^{\prime} \exp \left\{-i\left[Q_{x}(z) x^{2} / 2+Q_{y}(z) y^{2} / 2\right.\right. \\
& \left.\left.+S_{x}(z) x+S_{y}(z) y+P(z)\right]\right\} \\
& \times H_{m}\left\{\sqrt{2}\left[x-\delta_{x}(z)\right] / W_{x}(z)\right\} \\
& \times H_{n}\left\{\sqrt{2}\left[y-\delta_{y}(z)\right] / W_{y}(z)\right\}
\end{aligned}
$$

and the notation $\operatorname{Re}($ ) designates the real part of a complex function. Thus a given beam mode contains essentially three parts - a plane-wave factor, a Gaussian factor, and a Hermite-polynomial factor of complex argument. Though the beam modes are essentially TEM, the $z$ component of the field vector may be obtained from the Heaviside form of Maxwell's equations. ${ }^{12}$

The angular frequency, $\omega$; of the plane-wave factor is related to its wavelength by the equation $\omega=2 \pi c / \lambda$, where $c$ is the vacuum speed of light. The real constant $\nu$ is the angle of the linearly polarized field from the $x$ axis. The complex axial propagation constant is $k_{0}(z)=$ $2 \pi n_{0}(z) / \lambda+i \alpha_{0}(z)$, where $n_{0}(z)$ is the $z$-dependent axial index of refraction of the medium in which the light beam propagates. The function $\alpha_{0}(z)$ is the $z$-dependent axial gain coefficient. Since the electric field given in Eq. (3) is a solution of a linear differential equation, superposition may be used. Thus the total field may be a discrete or continuous sum of the modes in Eq. (3) with a variety of frequencies, polarizations, and field distributions.

The Gaussian factor in Eq. (4) contains the beam parameters $Q_{x}(z)$ and $Q_{y}(z)$, the displacement parameters $S_{x}(z)$ and $S_{y}(z)$, and the phase parameter $P(z)$. If the input and output planes are taken in a lossless medium (such as free space) or if the low gain (or loss) per wavelength approximation is made, then the beam parameters are related to the beam's spot size (beam width) $w_{x}$ and radius of phase curvature $R_{x}$ by

$$
\frac{Q_{x}}{\beta_{0}} \equiv \frac{1}{q_{x}}=\frac{1}{R_{x}}-i \frac{2}{\beta_{0} w_{x}^{2}},
$$

and the displacement parameters are related to the position $d_{x a}$ and the slope $d_{x a}^{\prime}$ of the beam by ${ }^{7}$

$$
\frac{S_{x}}{\beta_{0}}=-\frac{d_{x a}}{q_{x}}+d_{x a}^{\prime}=\left(-\frac{d_{x a}}{R_{x}}+d_{x a}^{\prime}\right)+i \frac{2 d_{x a}}{\beta_{0} w_{x}^{2}}
$$

Here and everywhere hereafter each $x$-subscripted equation implies a corresponding $y$-subscripted equation, which is not written for conciseness. As a complex equation, Eq. (6) represents two real equations with two unknowns. It is useful to have them in inverted form:

$$
\begin{aligned}
& d_{x a}=S_{x i} w_{x}^{2} / 2 \\
& d_{x a}^{\prime}=S_{x r} / \beta_{0}+\frac{S_{x i} w_{x}^{2}}{2 R_{x}}
\end{aligned}
$$

The real and imaginary parts of the phase parameter $P(z)$ represent axial phase and amplitude shifts.

The generalized beam matrix may be used to propagate the beam and displacement parameters through an astigmatic misaligned complex optical system. It may be written as $^{7}$

$$
\left(\begin{array}{c}
u_{x 2} \\
\left(1 / q_{x 2}\right) u_{x 2} \\
S_{x 2} u_{x 2}
\end{array}\right)=\left[\begin{array}{lll}
A_{x} & B_{x} & 0 \\
C_{x} & D_{x} & 0 \\
G_{x} & H_{x} & 1
\end{array}\right]\left(\begin{array}{c}
u_{x 1} \\
\left(1 / q_{x 1}\right) u_{x 1} \\
S_{x 1} u_{x 1}
\end{array}\right),
$$

where the subscripts 1 and 2 represent the input and output parameters of the optical element or system, respectively, and the matrix elements may each be complex. Optical systems may be analyzed by multiplication of the matrix representations of the optical elements in the reverse of the order in which those elements are encountered by the incident light beam.

The parameters $u_{x 1}$ and $u_{x 2}$ appearing in Eq. (9) are analogous in some respects to the input and output ray positions in ray optical models with real matrix elements. 
However, it should be emphasized that only ratios of the vector components in Eq. (9) have a direct physical meaning in the present complex formalism. Dividing the second row of Eq. (9) by its first row yields the Kogelnik transformation, which was originally known as the $A B C D$ law ${ }^{32}$ :

$$
\frac{1}{q_{x 2}}=\frac{C_{x}+D_{x} / q_{x 1}}{A_{x}+B_{x} / q_{x 1}}
$$

Similarly, the displacement transformation is obtained by division of the third row of Eq. (9) by its first row ${ }^{7}$ :

$$
S_{x 2}=\frac{S_{x 1}}{A_{x}+B_{x} / q_{x 1}}+\frac{G_{x}+H_{x} / q_{x 1}}{A_{x}+B_{x} / q_{x 1}}
$$

The $H_{m}$ and $H_{n}$ functions in Eq. (4) are Hermite polynomials whose arguments are, in general, complex. The first transformation of the parameters in this polynomial factor is ${ }^{33}$

$$
W_{x 2}^{2}=W_{x 1}^{2}\left(A_{x}+B_{x} / q_{x 1}\right)^{2}+4 i B_{x}\left(A_{x}+B_{x} / q_{x 1}\right) / k_{01}
$$

In material media $A_{x} D_{x}-B_{x} C_{x}=k_{0}(0) / k_{0}(z)$,

$$
\begin{aligned}
C_{x} & \equiv \mathrm{d} A_{x} / \mathrm{d} z \\
D_{x} & \equiv \mathrm{d} B_{x} / \mathrm{d} z \\
G_{x} & \equiv-\frac{1}{2} \int_{0}^{z} k_{1}\left(z^{\prime}\right) A_{x}\left(z^{\prime}\right) \mathrm{d} z^{\prime}, \\
H_{x} & \equiv-\frac{1}{2} \int_{0}^{z} k_{1}\left(z^{\prime}\right) B_{x}\left(z^{\prime}\right) \mathrm{d} z^{\prime},
\end{aligned}
$$

and the differential equation for $\delta_{x}$, the complex displacement of the polynomial term in Eq. (4) is ${ }^{34}$

$$
\frac{\mathrm{d} \delta_{x}}{\mathrm{~d} z}-\frac{1}{q_{x}(z)} \delta_{x}(z)=\frac{S_{x}(z)}{k_{0}(z)} .
$$

transformation [Eq. (15)] may also be used for off-axis Laguerre-Gaussian beam modes in misaligned complex optical systems. ${ }^{35}$

Though our analysis has dealt with complex-argument modes, there may also be interest in polynomial-Gaussian beam modes in which the arguments of the polynomials are real. As opposed to the complex-argument beam modes, these real-argument modes have simple spherical phase fronts. It is shown in Appendix A that the real-argument modes are a special case of the complexargument modes when the generalized beam matrix is purely real. However, real-valued beam matrices correspond to lossless optical systems (with the exception of a uniform thin lossy element such as a conventional partially reflecting uniform mirror). Thus the effect of nonuniform loss is to distort the spherical phase front of a polynomial-Gaussian beam. Real-argument modes are not eigenmodes of complex optical systems.

The Gaussian factor in Eq. (4) contains a phase parameter $P(z)$. It is difficult to integrate the corresponding phase parameter equation for a medium represented by an arbitrary generalized beam matrix. However, for an aligned medium, $G_{x}=0$ and $H_{x}=0$, and the phase parameter is ${ }^{35}$

$$
\begin{aligned}
P_{2}-P_{1}= & -\frac{i}{2} \ln \left(A_{x}+B_{x} / q_{x 1}\right)-\frac{i}{2} \ln \left(A_{y}+B_{y} / q_{y 1}\right) \\
& +\frac{i}{2}\left[m \ln \left(1+\frac{4 i}{k_{01} W_{x 1}^{2}} \frac{B_{x}}{A_{x}+B_{x} / q_{x 1}}\right)\right. \\
& \left.+n \ln \left(1+\frac{4 i}{k_{01} W_{y 1}^{2}} \frac{B_{y}}{A_{y}+B_{y} / q_{y 1}}\right)\right] \\
& -\frac{1}{2 k_{01}}\left(\frac{S_{x 1}{ }^{2} B_{x}}{A_{x}+B_{x} / q_{x 1}}\right)-\frac{1}{2 k_{01}}\left(\frac{S_{y 1}^{2} B_{y}}{A_{y}+B_{y} / q_{y 1}}\right)
\end{aligned}
$$

The real part of the right-hand side of this equation is the axial phase shift experienced by an input beam as it propagates through an optical system. If one is interested in the gain in the axial field magnitude, it may be more convenient to rewrite Eq. (16) as

$\frac{\exp \left(-i P_{2}\right)}{\exp \left(-i P_{1}\right)}=\frac{\left(1+\frac{4 i}{k_{01} W_{x 1}^{2}} \frac{B_{x}}{A_{x}+B_{x} / q_{x 1}}\right)^{m / 2}\left(1+\frac{4 i}{k_{01} W_{y 1}^{2}} \frac{B_{y}}{A_{y}+B_{y} / q_{y 1}}\right)^{n / 2}}{\left(A_{x}+B_{x} / q_{x 1}\right)^{1 / 2}\left(A_{y}+B_{y} / q_{y 1}\right)^{1 / 2}} \exp \left[\frac{i}{2 k_{01}}\left(\frac{S_{x 1}{ }^{2} B_{x}}{A_{x}+B_{x} / q_{x 1}}+\frac{S_{y 1}{ }^{2} B_{y}}{A_{y}+B_{y} / q_{y 1}}\right)\right]$.

It can be shown by direct substitution that the exact solution of Eq. (14) is

$$
\begin{aligned}
\delta_{x 2}= & \delta_{x 1}\left(A_{x}+B_{x} / q_{x 1}\right)+S_{x 1} B_{x} / k_{01} \\
& +\left(B_{x} G_{x}-A_{x} H_{x}\right) / k_{01}
\end{aligned}
$$

This new transformation generalizes a previous result ${ }^{35}$ to include the effects of misalignment. The intensity distributions of some low-order complex-argument modes are plotted in Fig. 1 of Ref. 34. Though there has been an emphasis here on Hermite-Gaussian modes, the field distribution in Eq. (3) can be expressed in terms of Laguerre-Gaussian functions, and this new

\section{MODE STABILITY}

A. Fundamental-Mode Perturbation Stability

The Gaussian mode of a resonator that contains only complex optical elements of the types mentioned above may be found from the self-consistency requirement that $q_{x}$ repeat after a round trip through the resonator. If the round-trip matrix elements are $A_{x}, B_{x}, C_{x}, D_{x}, G_{x}$, and $H_{x}$, the steady-state beam parameter $q_{x \infty}$ at the chosen reference plane in the chosen direction must be a solution of

$$
1 / q_{x \infty}=\frac{C_{x}+D_{x} / q_{x \infty}}{A_{x}+B_{x} / q_{x \infty}}
$$


If $B_{x} \neq 0$, then this is a quadratic equation having the two roots

$$
1 / q_{x \infty}=\frac{D_{x}-A_{x}}{2 B_{x}} \pm \frac{i}{B_{x}}\left[1-\left(\frac{A_{x}+D_{x}}{2}\right)^{2}\right]^{1 / 2},
$$

where the unimodularity condition $A_{x} D_{x}-B_{x} C_{x}=1$, which applies to all optical resonators and periodic optical systems, has been used.

Equation (19) is sometimes written in another form:

$$
A_{x}+B_{x} / q_{x \infty}=\exp \left( \pm i \theta_{x}\right)
$$

where

$$
\cos \theta_{x} \equiv \frac{A_{x}+D_{x}}{2} .
$$

The two solutions given in Eq. (19) are not necessarily acceptable in a practical situation, and sometimes neither solution is desirable. For example, if all the matrix elements are real and the condition $-1 \leq\left(A_{x}+D_{x}\right) / 2 \leq 1$ is not satisfied, then from Eq. (19) the beam parameter is also real. But from Eq. (5) the resulting spot size is infinite. Therefore one may impose on Eq. (19) a confinement condition that requires that the square of the spot size be positive and finite. However, confinement in one plane of an optical resonator does not guarantee confinement everywhere within the resonator. For example, an inverted Gaussian aperture $t=t_{0} \exp \left(x^{2} / w_{\mathrm{ga}, x}{ }^{2}\right)$ at one plane can transform a confined beam into an unconfined one, and a following ordinary Gaussian aperture can transform the beam back again. Furthermore, confinement for a given direction of propagation at a given plane does not necessarily imply confinement for the opposite direction of propagation at the same plane. Confinement at all planes within the resonator for light beams propagating in both directions is required for a confined mode. However, there are lasers that exhibit unconfined ring modes. ${ }^{36}$

For single-pass modes a similar self-consistency requirement exists for the position and the slope of the beam, and applying the condition $S_{x 1}=S_{x 2}=S_{x \infty}$ to Eq. (11) results in

$$
S_{x \infty}=\frac{G_{x}+H_{x} / q_{x \infty}}{A_{x}+B_{x} / q_{x \infty}-1} .
$$

The resulting position and slope of the mode may be garnered from Eqs. (7) and (8).

Though physically realizable modes need not be confined, they must be perturbation stable, as perturbations that are due to, for example, imperfections in instrumentation and alignment always exist. ${ }^{36}$ If the beam parameter is perturbed by $\delta_{q}^{\prime}$, then after a round trip the perturbation becomes $\delta_{q}^{\prime \prime}$. These perturbations may be related with the use of Eq. (10):

$$
\begin{aligned}
\frac{1}{q_{x \infty}}+\delta_{q}^{\prime \prime} & =\frac{C_{x}+D_{x}\left(1 / q_{x \infty}+\delta_{q}^{\prime}\right)}{A_{x}+B_{x}\left(1 / q_{x \infty}+\delta_{q}^{\prime}\right)} \\
& =\left(\frac{C_{x}+D_{x} / q_{x \infty}}{A_{x}+B_{x} / q_{x \infty}}\right)\left[\frac{1+\delta_{q}^{\prime} D_{x} /\left(C_{x}+D_{x} / q_{x \infty}\right)}{1+\delta_{q}^{\prime} B_{x}\left(A_{x}+B_{x} / q_{x \infty}\right)}\right]
\end{aligned}
$$

$$
\approx\left(\frac{C_{x}+D_{x} / q_{x \infty}}{A_{x}+B_{x} / q_{x \infty}}\right)\left(1+\frac{\delta_{q}^{\prime} D_{x}}{C_{x}+D_{x} / q_{x \infty}}-\frac{\delta_{q}^{\prime} B_{x}}{A_{x}+B_{x} / q_{x \infty}}\right) .
$$

The unimodularity condition $\left(A_{x} D_{x}-B_{x} C_{x}=1\right)$ may be used, and it follows that, for à small initial perturbation $\delta_{q}^{\prime}$

$$
\left|\frac{\delta_{q}^{\prime \prime}}{\delta_{q}^{\prime}}\right| \approx \frac{1}{\left|A_{x}+B_{x} / q_{x \infty}\right|^{2}} .
$$

Though it is assumed in Eq. (19) that $B_{x} \neq 0$, this result is valid even when $B_{x}=0$. Mode stability is assured if the perturbation damps after each round trip. This is the case if ${ }^{36}$

$$
F_{x s} \equiv\left|A_{x}+B_{x} / q_{x \infty}\right|>1 .
$$

If we apply this stability criterion to Eq. (20), it follows that the two solutions given in Eq. (19) have stability factors that are reciprocals of each other. Thus, when one solution is stable, the other is unstable. There is always one and only one stable solution except when $A_{x}+$ $D_{x}$ is purely real and less than 2 in absolute value, in which case both solutions are metastable.

A similar stability analysis may be performed for the displacement parameter:

$$
S_{x \infty}+\delta_{S}^{\prime \prime}=\frac{S_{x \infty}+\delta_{S}^{\prime}}{A_{x}+B_{x} / q_{x \infty}}+\frac{G_{x}+H_{x} / q_{x \infty}}{A_{x}+B_{x} / q_{x \infty}} .
$$

For a perturbation of any size

$$
\left|\frac{\delta_{S}^{\prime \prime}}{\delta_{S}^{\prime}}\right|=\frac{1}{\left|A_{x}+B_{x} / q_{x \infty}\right|}=F_{x s}{ }^{-1} .
$$

Thus the same condition for stability exists [relation (27)] for the displacement parameter $S_{x}$ as for the beam parameter $Q_{x}$. These stability results are independent of whether the system is misaligned or curved, since the stability factor is independent of $G_{x}$ and $H_{x}$.

\section{B. Hermite-Gaussian Mode Perturbation Stability}

In the previous subsection we considered the stability of a fundamental-mode Gaussian beam. For polynomialGaussian beams oscillation conditions must be applied to the complex spot size $W_{x}$ and the complex displacement parameter $\delta_{x}$. If the condition $W_{x 2}=W_{x 1} \equiv W_{x \infty}$ is applied to Eq. (12), then

$$
\begin{aligned}
W_{x \infty}^{2} & =\frac{4 i B_{x}\left(A_{x}+B_{x} / q_{x \infty}\right) / k_{0}}{1-\left(A_{x}+B_{x} / q_{x \infty}\right)^{2}} \\
& =\mp \frac{2 B_{x} / k_{0}}{\left\{1-\left[\left(A_{x}+D_{x}\right) / 2\right]^{2}\right\}^{1 / 2}},
\end{aligned}
$$

where Eqs. (20) and (21) have been used. Though the complex spot size has some features that are similar to those of the beam parameter, there is no corresponding confinement condition.

A similar analysis is used for the displacement parameter governed by Eq. (15), and if $\delta_{x 2}=\delta_{x 1}=\delta_{x \infty}$, then the steady-state complex displacement parameter is 


$$
\begin{aligned}
\delta_{x \infty} & =\frac{\left(S_{x \infty} B_{x}+B_{x} G_{x}-A_{x} H_{x}\right) / k_{0}}{1-\left(A_{x}+B_{x} / q_{x \infty}\right)} \\
& =\frac{\left[\left(A_{x}-1\right) H_{x}-B_{x} G_{x}\right]\left(A_{x}+B_{x} / q_{x \infty}\right) / k_{0}}{\left(A_{x}+B_{x} / q_{x \infty}-1\right)^{2}} \\
& =\frac{\left[B_{x} G_{x}-\left(A_{x}-1\right) H_{x}\right] / k_{0}}{2\left[1-\left(A_{x}+D_{x}\right) / 2\right]},
\end{aligned}
$$

where Eqs. (20) and (21) have been used.

To determine the conditions for stability of the complex spot size, we perturb the steady-state value as above,

$$
\begin{aligned}
W_{x \infty}^{2}+\delta_{W}^{\prime \prime}= & \left(W_{x \infty}^{2}+\delta_{W}^{\prime}\right)\left(A_{x}+B_{x} / q_{x \infty}\right)^{2} \\
& +4 i B_{x}\left(A_{x}+B_{x} / q_{x \infty}\right) / k_{0},
\end{aligned}
$$

and the magnitude of the perturbation becomes

$$
\left|\frac{\delta_{W}^{\prime \prime}}{\delta_{W}^{\prime}}\right|=\left|A_{x}+B_{x} / q_{x \infty}\right|^{2}=F_{x s}{ }^{2},
$$

even for large perturbations. Thus an input beam's complex spot size approaches the value given in Eq. (31) if $F_{x s}<1$.

We use a perturbation of the complex displacement parameter,

$$
\begin{aligned}
\delta_{x \infty}+\delta_{\delta}^{\prime \prime}= & \left(\delta_{x \infty}+\delta_{\delta}^{\prime}\right)\left(A_{x}+B_{x} / q_{x \infty}\right)+S_{x \infty} B_{x} / k_{0} \\
& +\left(B_{x} G_{x}-A_{x} H_{x}\right) / k_{0}
\end{aligned}
$$

to find the magnitude of the perturbation:

$$
\left|\frac{\delta_{\delta}^{\prime \prime}}{\delta_{\delta}^{\prime}}\right|=\left|A_{x}+B_{x} / q_{x \infty}\right|=F_{x s .} .
$$

Similar to the behavior of the complex spot size, an input beam's complex displacement parameter approaches the value given in Eq. (34) if $F_{x s}<1$. As with the complex spot size, if $F_{x s}<1$, then the complex displacement parameter is stable even for large perturbations. However, both of these criteria are different from stability criteria for the beam parameter and the displacement parameter of the Gaussian portion of the field, which were stable when $F_{x s}>1$. Thus there are no conditions for which the Gaussian factor and the Hermite factor are simultaneously stable for an arbitrary mode index.

\section{DISCUSSION}

In one approach to beam mode analysis an initial field configuration is assumed. This initial field is allowed to propagate through the resonator of interest many times, and the limiting output field profile becomes a mode of the resonator. Alternatively, oscillation conditions may be used to determine the modes of a resonator. In this latter case perturbation stability criteria must be used to determine if the mode is experimentally realizable. This latter approach has been developed here.

Optical systems of large aperture that contain any combination of the following elements may be represented by a generalized beam matrix: Gaussian transmission filters (and Gaussian variable-reflectivity mirrors) and exponential transmission filters (and exponential variablereflectivity mirrors), thin lenses, thin prisms, spherical mirrors, and any medium representable by Eq. (2) with input and output planes that may be flat or quadratically curved, tilted, or displaced. When the effect of saturation on the beam mode is ignored, a laser composed of these optical elements may be represented by an infinite periodic lens waveguide. The generalized beam matrix for a unit cell of the lens waveguide may be obtained by multiplication in reverse order of the matrix representations of the optical elements that make up the unit cell. The laser that corresponds to the lens waveguide will operate in the fundamental mode when the stability condition

$$
\begin{aligned}
F_{x s} & =\left|A_{x}+B_{x} / q_{x \infty}\right| \\
& =\left|\frac{A_{x}+D_{x}}{2} \pm i\left[1-\left(\frac{A_{x}+D_{x}}{2}\right)^{2}\right]^{1 / 2}\right|>1
\end{aligned}
$$

is satisfied. If the confinement condition, $w_{x}^{-2}>0$, is also satisfied, then the fundamental mode is Gaussian. When the stability condition is not satisfied, there is no mode that is stable.

It has been noted that the $\mathrm{TEM}_{00}$ and $\mathrm{TEM}_{10}$ modes have the same stability factor for aligned optical systems. ${ }^{37}$ This can be seen by examination of the field distributions. The electric-field distributions for these modes are

$$
\begin{aligned}
E_{00}^{\prime}(x, y, z)= & E_{00,0}^{\prime} \exp \left\{-i\left[Q_{x}(z) x^{2} / 2+Q_{y}(z) y^{2} / 2\right.\right. \\
& \left.\left.+S_{x}(z) x+S_{y}(z) y+P(z)\right]\right\}, \\
E_{10}^{\prime}(x, y, z)= & E_{00}^{\prime} \frac{E_{10,0}^{\prime}}{E_{00,0}^{\prime}} \frac{\sqrt{2}\left(x-\delta_{x}\right)}{W_{x 1}\left(A_{x}+B_{x} / q_{x 0}\right)},
\end{aligned}
$$

where Eqs. (4), (12), and (17) have been employed. In particular, $E_{10}^{\prime}(x, y, z)$ is independent of $W_{x 2}(z)$, and thus the beam does not suffer from the instability of the transformation of the complex spot size. If the system and the input beam are aligned, then $\delta_{x 2}(z) \equiv 0$, and it follows that the $\mathrm{TEM}_{00}$ and $\mathrm{TEM}_{10}$ modes have the same stability.

To investigate which of the two modes the laser operates in, we compare the power gain per pass of each mode:

$$
\begin{aligned}
G_{p, m n} & \equiv \frac{\operatorname{Power}_{m n}(z)}{\operatorname{Power}_{m n}(0)} \\
& =\frac{\int_{-\infty}^{\infty} \int_{-\infty}^{\infty} E_{m n}^{\prime *}(x, y, z) E_{m n}^{\prime}(x, y, z) \mathrm{d} x \mathrm{~d} y}{\int_{-\infty}^{\infty} \int_{-\infty}^{\infty} E_{m n}^{\prime *}(x, y, 0) E_{m n}^{\prime}(x, y, 0) \mathrm{d} x \mathrm{~d} y} .
\end{aligned}
$$

From Eqs. (40)-(42) it follows that the ratio of the power gains of the two modes is

$$
\frac{G_{p, 00}}{G_{p, 10}}=\frac{\left|E_{00,0}^{\prime}{ }^{2}\right|}{\left|E_{10,0}^{\prime}\right|} \frac{\left|W_{x 1}\right|^{2}}{w_{x 1}{ }^{2}}\left|A_{x}+B_{x} / q_{x 0}\right|^{2} .
$$

If both modes have the same initial power, then

$$
\frac{G_{p, 00}}{G_{p, 10}}=\left|A_{x}+B_{x} / q_{x \infty}\right|^{2}=F_{x s}{ }^{2},
$$

and the laser will operate in the fundamental mode since it has a larger gain per pass than the TEM 10 mode.

As a final note, the stability factor $F_{x s}$ is independent of $G_{x}$ and $H_{x}$. Thus transverse-mode selection within a 
laser is independent of misalignments or curvatures of the optical elements within the resonator. Furthermore, it is also independent of the presence of prisms or exponential apertures.

\section{CONCLUSION}

In a previous study the generalized beam matrix method was established to propagate fundamental Gaussian beams in misaligned complex optical systems. Here a new transformation was obtained so that the formalism may be applied to polynomial-Gaussian beams in these optical systems. An important application of the theory involves the investigation of the mode selection in lasers and periodic optical systems, and this problem was considered here. In particular, it was found that when the stability factor $F_{x s}>1$, the laser operates in the fundamental mode. If the confinement condition $w_{x}{ }^{-2}>0$ is also satisfied, then this mode is Gaussian. When $F_{x s}=1$, all the modes are metastable and the laser cannot discriminate between the modes. When $F_{x s}<1$, all the modes are unstable. In practice, they encounter a system aperture or other mode-mixing element that invalidates the generalized beam matrix characterization for the system. Paraxial misalignment has no effect on single-pass mode selection.

\section{APPENDIX A: MODES OF MISALIGNED LOSSLESS OPTICAL SYSTEMS}

In this appendix it is shown that in misaligned lossless optical systems the modes in which the arguments of the Hermite polynomials are complex and the usual realargument modes are identical. Thus the real-argument modes need not be considered independent of the complexargument modes but are merely a special case of the more general mode set.

For beam propagation in lossless optical systems $A_{x}$, $B_{x}, C_{x}, D_{x}, G_{x}, H_{x}$, and $k_{0}$ are all real. It has previously been shown that under these circumstances, when the input complex spot size $W_{1}=w_{1}$ (i.e., the input beam is a real-argument mode), the output complex spot size is real and equal to the conventional spot size at the output (i.e., $\left.W_{2}=w_{2}\right)^{38}$ Thus a real-argument mode input on-axis into an aligned lossless optical system remains a realargument mode.

Similar results apply to off-axis modes in misaligned lossless optical systems. In particular, if $\delta_{x 1}=d_{x a 1}$, then Eq. (6) may be substituted into Eq. (15), and it follows that

$$
\begin{aligned}
\delta_{x 2}= & d_{x a 1}\left(A_{x}+B_{x} / q_{x 1}\right)+\left(d_{x a 1}^{\prime}-d_{x a 1} / q_{x 1}\right) B_{x} \\
& +\left(B_{x} G_{x}-A_{x} H_{x}\right) / \beta_{0} \\
= & A_{x} d_{x a 1}+B_{x} d_{x a 1}^{\prime}+\left(B_{x} G_{x}-A_{x} H_{x}\right) / \beta_{0} \\
= & A_{x} d_{x a 1}+B_{x} d_{x a 1}^{\prime}+E_{x} \\
= & d_{x a 2},
\end{aligned}
$$

and the displacement of the polynomial factor remains identical to the Gaussian factor amplitude displacement. Thus, when the oscillation conditions are applied to laser resonators represented by real beam matrices, the polynomial-Gaussian modes of complex argument are indistinguishable from the corresponding real-argument modes. It may therefore be concluded that the real-argument modes need not be considered separately from the complex-argument modes and that oscillation conditions may always be applied to the complex-argument modes.

\section{ACKNOWLEDGMENT}

This work was supported in part by the National Science Foundation under grant ECS-9014481.

*Present address, Department of Physics and Astronomy, Murray State University, Murray, Kentucky 42071-0009.

\section{REFERENCES}

1. N. G. Vakhimov, "Open resonators with mirrors having variable reflection coefficients," Radio Eng. Electron. Phys. (USSR) 10, 1439-1446 (1965).

2. H. Zucker, "Optical resonators with variable reflectivity mirrors," Bell Syst. Tech. J. 49, 2349-2376 (1970).

3. J. A. Arnaud, "Optical resonators in the approximation of Gauss," Proc. IEEE 62, 1561-1570 (1974).

4. L. W. Casperson and S. D. Lunnam, "Gaussian modes in high loss laser resonators," Appl. Opt. 14, 1193-1199 (1975).

5. A. Yariv and P. Yeh, "Confinement and stability in optical resonators employing mirrors with Gaussian reflectivity tapers," Opt. Commun. 13, 370-374 (1975).

6. U. Ganiel, A. Hardy, and Y. Silberberg, "Stability of optical laser resonators with mirrors of Gaussian reflectivity profiles, which contain an active medium," Opt. Commun. 14, 290-293 (1975).

7. A. A. Tovar and L. W. Casperson, "Generalized beam matrices: Gaussian beam propagation in misaligned complex optical systems," J. Opt. Soc. Am. A 12, 1522-1533 (1995).

8. A. A. Tovar and L. W. Casperson, "Gaussian beam optical systems with high gain or high loss media," IEEE Trans. Microwave Theory Tech. 43, 1857-1862 (1995).

9. L. W. Casperson and S. J. Sheldrake, "Beam deflection and isolation in laser amplifiers," Opt. Commun. 12, 349-353 (1974).

10. H. Kogelnik, "On the propagation of Gaussian beams of light through lenslike media including those with a loss or gain variation," Appl. Opt. 4, 1562-1569 (1965).

11. L. W. Casperson and A. Yariv, "The Gaussian mode in optical resonators with a radial gain profile," Appl. Phys. Lett. 12, 355-357 (1968).

12. L. W. Casperson, "Gaussian light beams in inhomogeneous media," Appl. Opt. 12, 2434-2441 (1973).

13. N. McCarthy and P. Lavigne, "Large-size Gaussian mode in unstable resonators using Gaussian mirrors," Opt. Lett. 10, 553-555 (1985).

14. P. Lavigne, A. Parent, D. Pascale, and N. McCarthy, "A compact wide-aperature single-mode $\mathrm{TE}-\mathrm{CO}_{2}$ laser with a low chirp rate," IEEE J. Quantum Electron. QE-22, 2200-2203 (1986).

15. D. V. Willetts and M. R. Harris, "Output characteristics of a compact $1 \mathrm{~J}$ carbon dioxide laser with a Gaussian reflectivity mirror," IEEE J. Quantum Electron. 24, 849-855 (1988).

16. P. Lavigne, N. McCarthy, A. Parent, and K. J. Snell, "Laser mode control with variable reflectivity mirrors," Can. J. Phys. 66, 888-895 (1988).

17. S. De Silvestri, P. Laporta, V. Magni, and O. Svelto, "Unstable laser resonators with super-Gaussian mirrors," Opt. Lett. 13, 201-203 (1988).

18. K. J. Snell, N. McCarthy, M. Piche, and P. Lavigne, "Single transverse mode oscillation from an unstable resonator $\mathrm{Nd}$ :YAG laser using a variable reflectivity mirror," Opt. Commun. 65, 377-382 (1988).

19. S. De Silvestri, P. Laporta, V. Magni, G. Valentini, and G. Cerullo, "Comparative analysis of Nd:YAG unstable 
resonators with super-Gaussian variable reflectance mirrors," Opt. Commun. 77, 179-184 (1990).

20. S. De Silvestri, V. Magni, O. Svelto, and G. Valentini, "Lasers with super-Gaussian mirrors," IEEE J. Quantum Electron. 26, 1500-1509 (1990).

21. J. J. Kasinski, W. Hughes, D. DiBiase, P. Bournes, and R. Burnham, "One joule output from a diode-array-pumped Nd:YAG laser with side-pumped rod geometry," IEEE J. Quantum Electron. 28, 977-985 (1992).

22. D. M. Tratt, "Optimizing coherent lidar performance with graded-reflectance laser resonator optics," Appl. Opt. 31, 4233-4239 (1992).

23. K. L. Webster and C. C. Sung, "Mode-medium instability and its correction with a Gaussian-reflectivity mirror," Appl. Opt. 31, 319-328 (1992).

24. See, for example, P. Lavigne, N. McCarthy, and J.-G. Demers, "Design and characterization of complementary Gaussian reflectivity mirrors," Appl. Opt. 24, 2581-2586 (1985).

25. W. R. Bennett, "Inversion mechanisms in gas lasers," Appl. Opt. Suppl. 2, Chemical Lasers, pp. 3-33 (1965).

26. B. N. Perry, P. Rabinowitz, and M. Newstein, "Wave propagation in media with focused gain," Phys. Rev. A 27, 1989-2002 (1983).

27. See the introduction of S. Zaidi and D. L. MacFarlane, "Mode evolution of optical resonators with a radial gain profile," Phys. Rev. A 47, 588-596 (1993), and references therein.

28. F. R. Nash, "Mode guidance parallel to the junction plane of double-heterostructure GaAs lasers," J. Appl. Phys. 44, 4696-4707 (1973).

29. N. McCarthy and P. Lavigne, "Optical resonators with Gaussian reflectivity mirrors: misalignment sensitivity," Appl. Opt. 22, 2704-2708 (1983).

30. N. McCarthy and P. Lavigne, "Optical resonators with Gaussian reflectivity mirrors: output beam characteristics," Appl. Opt. 23, 3845-3850 (1984).

31. N. McCarthy and M. Morin, "High-order transverse modes of misaligned laser resonators with Gaussian reflectivity mirrors," Appl. Opt. 28, 2189-2191 (1989).

32. H. Kogelnik, "Imaging of optical modes - resonators with internal lenses," Bell Syst. Tech. J. 44, 455-494 (1965).

33. M. Nazarathy, A. Hardy, and J. Shamir, "Generalized mode propagation in first-order optical systems with loss or gain," J. Opt. Soc. Am. 72, 1409-1420 (1982).

34. L. W. Casperson, "Beam modes in complex lenslike media and resonators," J. Opt. Soc. Am. 66, 1373-1379 (1976).

35. A. A. Tovar and L. W. Casperson, "Off-axis complexargument polynomial-Gaussian beams in optical systems," J. Opt. Soc. Am. A 8, 60-68 (1991).

36. L. W. Casperson, "Mode stability of lasers and periodic optical systems," IEEE J. Quantum Electron. QE-10, 629-634 (1974).

37. A. E. Siegman, Lasers (University Science, Mill Valley, Calif., 1986), p. 837.

38. Ref. 37 , pp. $798-801$. 